\title{
BMJ Open Ephedrine as add-on therapy for patients with myasthenia gravis: protocol for a series of randomised, placebo-controlled $\mathbf{n}$-of- $\mathbf{1}$ trials
}

Charlotte Vrinten, ${ }^{1,2}$ Alexander F Lipka, ${ }^{3}$ Erik W van Zwet, ${ }^{4}$ Kirsten J M Schimmel, ${ }^{5}$ Martina C Cornel, ${ }^{2}$ Marja R Kuijpers, ${ }^{6}$ Yechiel A Hekster, ${ }^{7}$ Stephanie S Weinreich, ${ }^{2}$ Jan J G M Verschuuren ${ }^{3}$

To cite: Vrinten C, Lipka AF, van Zwet EW, et al. Ephedrine as add-on therapy for patients with myasthenia gravis: protocol for a series of randomised, placebocontrolled n-of- 1 trials. BMJ Open 2015;5:e007863. doi:10.1136/bmjopen-2015007863

- Prepublication history for this paper is available online To view these files please visit the journal online (http://dx.doi.org/10.1136/ bmjopen-2015-007863).

Received 4 February 2015 Revised 8 April 2015 Accepted 12 April 2015

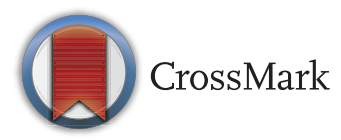

For numbered affiliations see end of article.

Correspondence to Charlotte Vrinten; c.vrinten@ucl.ac.uk

\section{ABSTRACT}

Introduction: Myasthenia gravis (MG), a rare neuromuscular disease, is often initially treated using acetylcholinesterase inhibitors. Patients who do not respond adequately depend on the use of corticosteroids or other immunosuppressive medication, but these may have serious side effects. Clinical observations suggest that ephedrine can diminish, postpone or even prevent the need for immunosuppressive therapy when added to acetylcholinesterase inhibitors or low-dose prednisone. In the Netherlands, ephedrine is not licensed for MG nor is reimbursement guaranteed. MG is a rare condition, and ephedrine might be indicated only in a subset of patients. Thus, randomised controlled trials comparing large groups are difficult to conduct. We, therefore, aim to aggregate data from a small series of n-of- 1 trials (also known as single patient trials) to assess the effect of ephedrine as add-on treatment for MG.

Methods and analysis: Single-centre, placebocontrolled, double-blind, randomised, multiple crossover n-of-1 studies in 4 adult patients with generalised MG who show inadequate improvement on pyridostigmine and/or immunosuppressive drugs. Each n-of- 1 trial has 3 cycles of two 5 -day intervention periods. Treatment: $25 \mathrm{mg}$ ephedrine or placebo, twice daily. Main outcome measure: Quantitative Myasthenia Gravis (QMG) test. Statistical analysis: fixed effects linear model for QMG for all patients combined. Secondary outcome measures: Clinical: effects on MGComposite and MG-Activities of Daily Living (MG-ADL) scales; QMG at individual level; adverse events.

Acceptability of trial design: number of patients eligible and enrolled; number of treatment cycles completed; patients' and caregivers' experiences.

Ethics and dissemination: This study was approved by the Medical Ethics Committee of Leiden University Medical Center, No. P14.108. Results of the trial will be reported in a peer-reviewed publication. Regulatory stakeholders will comment on the suitability of the trial for market authorisation and reimbursement purposes. Trial registration number: This study is registered under EudraCT number 2014-001355-23, protocol no. 40960, V.1.0, registration date 27 March 2014.
Strengths and limitations of this study

Series of prospective, randomised, placebocontrolled trials for a rare disease.

- 'Level 1' evidence of add-on treatment effect of ephedrine in myasthenia gravis.

- Optional, 6-month open-label extension phase after prospective trial.

- Harms and benefits assessed, as well as acceptability of this type of trial.

Potential limitations include limited generalisability due to small sample size.

\section{INTRODUCTION}

Myasthenia gravis (MG) is an autoimmune disorder in which antibodies are directed against components of the neuromuscular junction, resulting in an impairment of neuromuscular transmission. MG is a rare disorder, with a prevalence of about 78 per million $^{1-3}$ and an incidence of 4-6 per million. ${ }^{3}{ }^{4}$ Clinically, MG is characterised by fluctuating muscle weakness in various muscle groups and fatigability, often resulting in marked impairment in activities of daily living (ADL). Muscle weakness and fatigability are often more pronounced at the end of the day, after exercise, during warm weather or during illness. ${ }^{5-8}$

Many patients respond well to first-line treatment with acetylcholinesterase inhibitors (AChEIs), such as pyridostigmine, which inhibit the activity of the enzyme that breaks down the neurotransmitter acetylcholine and thus, enhances neuromuscular transmission. ${ }^{69}$ This treatment is well tolerated by most patients. If symptoms can no longer be controlled with AChEIs, the second step in MG treatment consists of immunosuppression with high doses of corticosteroids (prednisone), azathioprine, or other immunosuppressant 
agents; ${ }^{6}$ however, these can give rise to moderate or serious side effects.

Anecdotal evidence and our clinical observations suggest that some patients with MG can avoid high-dose immunosuppressive treatment by using ephedrine as add-on treatment to AChEIs or low doses of prednisone. Ephedrine is a sympathomimetic agent which acts on the adrenergic receptors. ${ }^{10}$ Its mechanism of action in MG has been investigated, ${ }^{11-14}$ but is not well understood. ${ }^{1516}$ One study showed that ephedrine increases the quantal content of the endplate potential as well as the probability of quantal release of acetylcholine in an in vitro canine model, but only at concentrations that would not be reached in patients. ${ }^{14}$ Another study suggested that ephedrine improves neuromuscular transmission by stimulating $\beta_{2}$-adrenergic receptors and stabilising the structure of the neuromuscular junction. ${ }^{16}$ Clinically, the onset of the effect is usually seen within hours to days, although some studies in congenital MG suggest that it may take weeks to months before the full effect is reached in some patients. ${ }^{17} 18$ The effects of ephedrine in patients with MG have not been studied extensively, ${ }^{19}$ but it appears to be well tolerated (personal observations by JJGMV). Side effects consist of adrenergic effects, such as palpitations, restlessness, insomnia, headache and nausea, which are most likely dose dependent. ${ }^{19}$

\section{Rationale for the trial}

Although anecdotal evidence ${ }^{20-22}$ and our clinical observations suggest that some patients with MG may benefit from ephedrine, a recent Cochrane systematic review showed that its effects have never been formally assessed in randomised controlled, clinical trials (RCTs). ${ }^{19}$ This lack of evidence hinders market approval of ephedrine for MG and may also hinder its reimbursement.

MG is a rare disease, and there are only a small number of patients who might benefit from add-on treatment with ephedrine. It is, therefore, difficult to conduct group-RCTs to determine its effectiveness. $N$-of-1 trials (also known as single patient trials or n-of-1 RCTS) may provide a way of gathering scientifically robust evidence of effectiveness in small patient groups. N-of-1 trials are randomised, controlled, multiple crossover trials in single patients, in which data are contributed over several treatment cycles. Evidence from several n-of-1 trials can be aggregated to produce population treatment effect estimates. ${ }^{23-25}$ Given the multiple crossover character of n-of-1 trials (ie, each patient acts as their own control and contributes multiple cycles of data), the number of patients needed in n-of- 1 trials (ie, sample size) to estimate the population treatment effect is much reduced compared with group-RCTs ${ }^{26}$ and thus makes this type of trial very suitable to conduct research in small patient populations. ${ }^{27} 28$

\section{Aim and objectives}

We aim to conduct a series of n-of- 1 trials to obtain scientifically robust ('level 1') evidence ${ }^{29}$ of benefits and harms of add-on treatment with ephedrine for autoimmune MG, followed by an optional open-label extension phase for patients who decide to continue treatment with ephedrine. The primary endpoint is the study population treatment effect of ephedrine compared with placebo on muscle strength and endurance, as measured by the Quantitative Myasthenia Gravis (QMG) score, when ephedrine is added to regular treatment with pyridostigmine, either alone or in combination with stable low-dose prednisone or immunosuppressive treatment.

Key secondary objectives include:

- Describing the effects of add-on treatment with ephedrine on secondary outcome measures, including the MG-Composite $^{30} 31$ and MG-Activities of Daily Living (MG-ADL) $;^{32}$

- Determining the effect of add-on treatment with ephedrine for each patient individually;

- Recording any adverse effects of add-on treatment with ephedrine.

Other secondary objectives include:

- Evaluating the acceptability of the trial design by exploring the perspectives of patients, physicians, and pharmacists on the use of n-of- 1 trials, and by recording the number of people eligible for the study, the number of people who enrol, complete the multiple crossover phase of the trial, and elect to continue treatment with ephedrine during the open-label extension phase;

- Comparing immediate and long-term effects of ephedrine (for patients participating in the open-label extension phase).

Until now, n-of-1 trials have generally been conducted to guide individual therapeutic decisions. ${ }^{33} 34$ The current series of trials is part of a project which aims to create more knowledge about the suitability of n-of- 1 trials for regulatory decisions such as reimbursement and marketing authorisation. Regulatory and patient stakeholders were, therefore, consulted on the design of the protocol, and both will contribute to dissemination of trial results (see 'Dissemination of results' and 'Discussion' sections).

In addition, theoretical and actual benefits and burdens for n-of-1 trial participants have been described for more common disorders such as osteoarthritis and Attention Deficit Hyperactivity Disorder (ADHD). ${ }^{33-36}$ This study will explore whether benefits and burdens of n-of-1 trials conducted for rare diseases (where there are often fewer treatment options available) are similar to those for more common diseases. The experiences of physicians and pharmacists involved in the trial will be compared with experiences of their counterparts in a routine n-of- 1 trial service in Australia. ${ }^{35}$

\section{METHODS AND ANALYSIS}

Study design and duration

The study consists of a small series of n-of- 1 trials followed by an optional open-label extension phase. Each 
n-of-1 trial is a randomised, placebo-controlled, physician-blinded and patient-blinded, multiple crossover trial in a single patient. The n-of- 1 trials will consist of three cycles of 2 weeks duration, each consisting of one period of ephedrine treatment and one period of placebo treatment. The order of the treatment periods will be randomised. This 6-week multiple crossover phase will be followed by a 4-week evaluation phase, during which data analysis of the multiple crossover phase, feedback of results to the patient and their treating physician, and evaluation of the trial will take place. Thus, each n-of-1 trial will take 10 weeks to complete. The optional open-label extension phase which follows the n-of- 1 trial will be a further 6 months. The design of the trial is presented in figure 1.

\section{Study setting}

The first doses of the two periods of the first cycle will be administered during an inpatient visit. All clinical measures and tests (eg, QMG, ECG, blood tests) will be performed during outpatient visits. All other study medication as well as self-report questionnaire measures will be administered in the home setting. The 6-month open-label extension phase will be conducted in the home setting, with outpatient follow-up visits at 2, 4 and 6 months.

\section{Study population}

Adult patients with generalised autoimmune MG, who are being treated at Leiden University Medical Center (LUMC). Inclusion and exclusion criteria are described in box 1 .

\section{Sample size}

The QMG test results in a numerical score between 0 and 39, with higher scores indicating more severe disease. The estimated SD of repeated measures for a single person is 2.95 based on our clinical observations and an earlier trial. ${ }^{37}$ An average decrease in QMG score of 3.5 points is considered clinically relevant. ${ }^{38} 39$ A linear model with fixed effects for treatment and patient will be fitted to the repeated QMG measurements. Our sample size computations are based on the test of significance of the treatment effect in this model (see 'Analysis' section). Monte Carlo simulations were used to compute the power with three cycles (ie, 6 measurements) per patient for 3,4 , and 5 patients, which was $0.648,0.772$ and 0.862 , respectively. In light of the availability of eligible patients, time and resources, we chose a sample size of 4 patients, achieving approximately $77 \%$ power.

\section{Blinding, treatment allocation, and randomisation}

Patients and physicians will be blinded to the treatment sequence during the multiple crossover phase of the n-of-1 trial. Randomisation will be carried out for each individual n-of-1 trial by the dispensing hospital pharmacy to ensure patient and physician blinding, and will be performed in MS Excel. Ephedrine and placebo treatments will be randomised in a $1: 1$ ratio per cycle over the three cycles of the n-of- 1 trial (eg, AB-BA-BA). Unblinding will occur when a participant has completed the multiple crossover phase of the trial or in the case of a serious adverse event (SAE) which cannot be treated without knowing which treatment the patient was receiving (see also 'Safety Evaluation' section).

\section{Interventions and dosing schedule}

During the entire trial, treatment with pyridostigmine, low-dose prednisone and steroid-sparing drugs, such as azathioprine, will be continued by the patient according to their prestudy treatment schedule. For the n-of- 1 trial, oral add-on treatment with ephedrine $25 \mathrm{mg}$ twice daily will be compared with add-on treatment with a placebo twice daily. To this purpose, commercial tablets of $50 \mathrm{mg}$ ephedrine will be divided into two equal halves, and repackaged in a non-transparent capsule. For the placebo, a placebo tablet will be repackaged in a nontransparent capsule. The divided $50 \mathrm{mg}$ commercial tablets comply with the European Pharmacopoeia (Ph. Eur.) test for uniformity of mass for subdivided parts. For the open-label extension phase, the ephedrine tablets will be administered as such.

Any medication for coexisting conditions will be continued as before the trial, as well as any other interventions aimed at improving symptoms of MG (eg, physical therapy). Accountability will be assessed by the dispensing pharmacy, who will maintain records of drugs dispensed. Participant compliance will be assessed by the physician who will ask participants to return all unused study medication and will record pill count. Prednisone and cholinesterase inhibitor dosing will be documented as concomitant medication.

\section{Rescue medication}

The study protocol does not specify any rescue medication to treat side effects of ephedrine treatment because in our experience these are usually mild at the dose used in this study. In case of SAEs, the patient may be withdrawn from the trial and appropriate treatment will be started.

\section{Trial procedures}

Appropriately trained medical staff will carry out the screening for inclusion and exclusion criteria, including an ECG recording, and enrolment of participants into the n-of-1 trials. Baseline QMG will be measured and a standard neurological examination will be conducted. To standardise QMG measurements, all assessments will be conducted at a predefined time-interval after the last dose of study medication.

N-of-1 trial: multiple crossover phase and evaluation phase

The n-of- 1 trial consists of a double-blinded multiple crossover phase (weeks 1-6) and an evaluation phase (weeks 7-10; see figure 1). The multiple crossover phase 
Figure 1 Design of the study. Treatment periods ( $A$ and $B$ ) will be randomised. Figure not to scale.

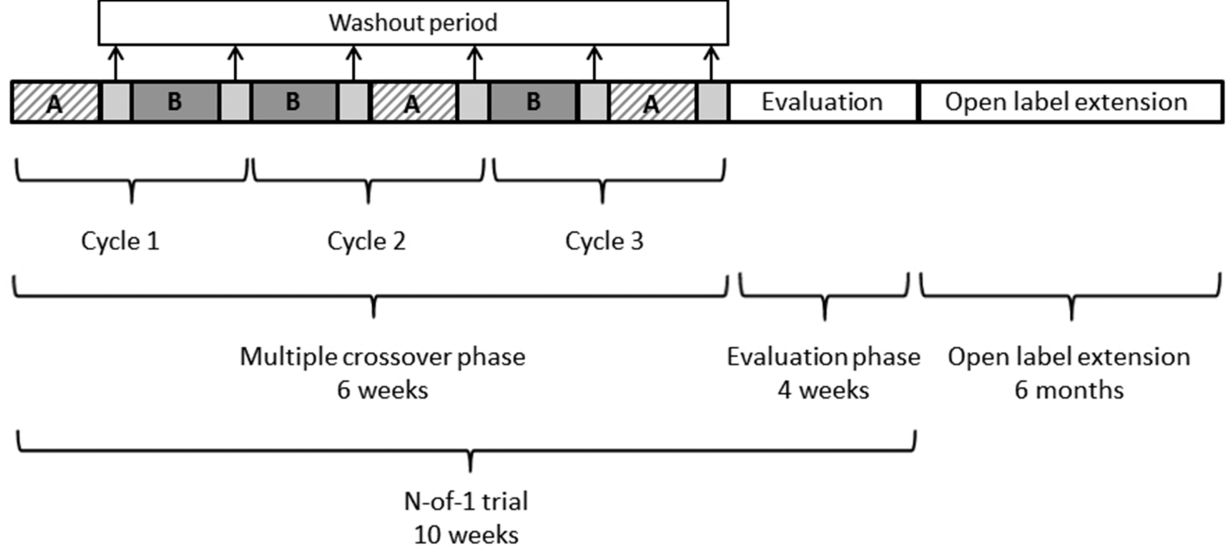

consists of three cycles with two 1-week treatment periods: one period of 5 days (Monday-Friday) when ephedrine is taken as add-on treatment, and one 5-day period (Monday-Friday) when a placebo is taken. The 5-day treatment periods are followed by a 2-day wash-out period (Saturday and Sunday) to prevent carry-over effects. A 2-day wash-out is considered adequate because of the short half-life of ephedrine of approximately $6 \mathrm{~h}^{40}$

On the first day of the first two periods (cycle 1), study medication will be administered in hospital to

\section{Box 1 Inclusion and exclusion criteria}

\section{Inclusion criteria}

- Adult patients with a diagnosis of generalised myasthenia gravis (MG), based on clinical signs or symptoms suggestive of generalised MG and confirmed by a positive serological test for acetylcholine receptor (AChR) antibodies

- No adequate improvement of myasthenic symptoms with pyridostigmine, either alone or in combination with low-dose (maximum $15 \mathrm{mg} /$ day) prednisone or other immunosuppressive drugs

- Stable dosages of pyridostigmine, prednisone or other immunosuppressive drugs for at least 6 weeks prior to trial enrolment

Exclusion criteria

- Purely ocular myasthenia

- Ephedrine was not tolerated in the past or is contraindicated due to myocardial ischaemia; cardiac arrhythmia; inherited prolonged QT syndrome or prolonged QT interval; angleclosure glaucoma; psychiatric treatment; hypertension; poorly regulated diabetes; prostatic hypertrophy; thyrotoxicosis; or relevant drug interactions (monoamine oxidase (MAO) inhibitors, $\alpha$ and $\beta$ blockers)

- Prednisone dose of $>15 \mathrm{mg} /$ day, recent (<3 months) or regular intravenous immunoglobulin or plasma exchange

- Myasthenic crisis in the past 3 months

- Thymectomy in the past 6 months, or scheduled to take place during the $n$-of- 1 trial

- Inability to fill out the study questionnaires, be interviewed in Dutch, undergo the trial's test procedures, give informed consent, or any other reason which renders the patient unsuitable to participate monitor adverse reactions. Study medication will be taken at home on all other days, with an outpatient hospital visit at the end of each treatment period. Treatment with pyridostigmine, low-dose prednisone or steroid-sparing drugs will be continued as before the trial, with a fixed dosing schedule.

The QMG score will be assessed by a neurologist or research nurse at baseline and during hospital visits at the end of each treatment period. The QMG has often been used in clinical trials and was recommended as outcome measure for all prospective research in MG by the Myasthenia Gravis Foundation of America (MGFA) ${ }^{41}$ Secondary outcomes that will be measured during this hospital visit include the MG-Composite ${ }^{30} 31$ and the MG-ADL ${ }^{32}$ to reflect recent recommendations by the MGFA. ${ }^{42}$ The effect of blinding will also be assessed during the hospital visit at the end of each cycle by recording which treatment the patient and physician believed was administered during each period.

Potential adverse effects of ephedrine will be monitored as follows: vital signs will be measured 30, 60 and 120 min after administration of study medication on the first day of periods 1 and 2. In addition, an ECG will be recorded $60 \mathrm{~min}$ after medication is administered on these days, and also during the hospital visit in weeks 1 , 2 and 6. Screening blood tests (haematology, liver and renal function tests) will be performed during hospital visits in weeks 1, 2 and 6 . Finally, a self-report symptom questionnaire will measure adverse effects on the first day of periods 1 and 2, as well as on the second and fourth day of every treatment period.

Following completion of the multiple crossover part of the trial, the results of each n-of-1 trial will be analysed by the researchers and a report will be drafted for each patient individually (weeks 7 and 8). This report, which will unblind patient and physician, will be made available to the treating physician to guide clinical decisionmaking and to discuss with the patient during an outpatient visit in week 8 . The resulting treatment decision will be recorded. In addition, the number of eligible patients, patients recruited, patients who completed their n-of- 1 trial, the total number of completed 
cycles, and the number of patients who decided to participate in the open-label extension phase will be recorded to evaluate the acceptability of this type of trial.

In addition to the quantitative evaluation of the acceptability of this type of trial, acceptability will also be evaluated qualitatively by exploring the experiences of patients, physicians and pharmacists in the trial. Semistructured telephone interviews of approximately 30 min will be conducted with the patient (week 9 or 10). The topics for the interview schedule were derived from methodological (theoretical) benefits and burdens of n-of-1 trials, ${ }^{24}$ published studies on experiences of patients and carers with n-of- 1 trials, ${ }^{33} 35$ and processrelated aspects of the trial at hand. The experiences of physicians and pharmacists involved in the trial will be explored quantitatively by registering the time they spend on the trial, as well as qualitatively from attending project meetings.

\section{Optional open-label extension phase (months 3-8)}

Participants who choose to enter the open-label extension phase will be followed up for 6 months to explore the long-term effects of add-on treatment with ephedrine. This will include hospital visits at 2, 4, and 6 months as part of regular clinical care, during which a standardised neurological examination, QMG, MG-Composite, and MG-ADL will be assessed and any adverse effects will be recorded.

\section{Safety evaluation}

\section{Adverse events}

Adverse events (AEs) and SAEs are defined according to the Dutch Medical Research Involving Human Subjects Act 2006. AEs will be monitored throughout the n-of-1 trial. All SAEs and Suspected Unexpected Serious Adverse Reactions (SUSARs) occurring during the study and either reported spontaneously or as part of the $\mathrm{AE}$ monitoring will be followed up by the principal investigator and will be reported separately to the Medical Ethics Committee (MEC). Unblinding will occur if there is reason to believe a SAE or SUSAR was due to the study medication and if the patient cannot be treated without knowing which treatment they were receiving. Unblinding and the reason for unblinding will be recorded. All AEs, SAEs and SUSARs will be followed up until they have abated or a stable situation has been reached.

\section{Removal from the trial and replacement of participants}

Participants will be removed from the study if informed consent is withdrawn. The investigator can decide to withdraw a participant from the n-of-1 trial for urgent medical reasons. Specific criteria for withdrawal of individual participants consist of:

- The occurrence of a SAE or SUSAR;

- A myasthenic crisis or worsening of symptoms requiring any treatment other than the trial medication;
- A need to undergo thymectomy before the end of a cycle in the multiple crossover phase of the trial.

Participants who are withdrawn from the study will be followed up until their planned end of the n-of- 1 trial to assess AEs of the trial medication. The cycles completed before a participant's withdrawal from the study will be analysed as part of the trial. Participants who fail to complete a single cycle of the multiple crossover phase will be replaced.

\section{Premature termination of the study}

The study will be terminated prematurely if SAEs occur that are deemed in all likelihood to be caused by the trial medication.

\section{Analysis}

Effect of add-on ephedrine compared with placebo on muscle strength and endurance for all patients

The average QMG scores for baseline, placebo and ephedrine periods of the multiple crossover phase will be described for all n-of-1 trials combined. A linear model for QMG score with fixed effects for treatment and patient will be fitted for all patients in the trial combined. All treatment effects for the study population will be tested at the 0.05 level.

If we can demonstrate a significant treatment effect in the study population, we conclude that, on average, ephedrine has an effect on the four patients enrolled in the study. If this is the case, we will proceed to test if there is a significant treatment effect in the population from which the four patients were sampled. To this end, we will fit a linear mixed model for QMG score with fixed effects for treatment and patient, and also a random treatment effect.

\section{Effects on secondary outcome measures}

For the secondary outcome measures, the average baseline score and the average scores for the ephedrine and placebo periods during the multiple crossover phase will be described for all n-of-1 trials combined.

\section{Effect of add-on treatment with ephedrine for each patient individually}

For each individual patient, QMG scores, scores for secondary outcome measures, and adverse effects reported during the ephedrine and placebo periods will be described in an individual patient report. This report will be discussed by the patient and their physician during the evaluation phase of the n-of- 1 trial and may be used to guide individual therapeutic decisions. All data from completed cycles will be used for the analyses of treatment effects. Given the small sample size, no additional analyses at group level, such as interim or subgroup analyses, are planned.

\section{Adverse effects, study inclusion and views of patients, physicians, and pharmacists}

A descriptive report will list the number of patients who experienced adverse effects during the placebo and 
ephedrine periods of the multiple crossover phase of the n-of- 1 trials, as well as the type of adverse effect. This includes both self-reported adverse effects and those measured by vital signs, ECG and blood test monitoring. The number of patients eligible for the trial, as well as the number of patients who enrolled in the trial, who completed the trial, and who opted to proceed with the open-label extension afterwards will be described. A qualitative analysis will explore the experiences of patients, physicians and pharmacists with the n-of- 1 trial, including patient satisfaction. This evaluation will also include a description of the average time cost of the n-of-1 trial for the physicians and pharmacists.

\section{Long-term effects of ephedrine}

Baseline QMG, MG-Composite and MG-ADL scores, and the scores measured during the n-of- 1 trials will be compared with the scores for these measures during the open-label extension phase at 2, 4 and 6 months.

\section{ETHICS AND DISSEMINATION Ethics approval}

Any amendments to the study protocol will be recorded and submitted to the MEC, with substantial amendments submitted for approval prior to implementation. An annual progress report, including information on the date of inclusion of the first participant; the total number of participants who have been included and completed the study; safety and AEs; and amendments to the protocol will be submitted annually and within 3 months of the end of the study (or within 15 days if the study is terminated prematurely). A final study report with its results and any publications or abstracts of the study will be submitted to the MEC and the Competent Authority within 1 year of the end of the study. Since AEs are strictly monitored as outlined earlier and the duration of blinding is only short (6 weeks during the multiple crossover phase of the n-of-1 trials), no additional data monitoring committee will be implemented.

This study is registered under EudraCT number 2014-001355-23, protocol no. 40960, V.1.0, registration date 27 March 2014. Registration details from the WHO Trial Registration Data Set can be found in table 1. The study will be conducted in accordance with the principles of the Declaration of Helsinki (2008) and in accordance with the Dutch Medical Research Involving Human Subjects Act and Good Clinical Practice guidelines.

\section{Recruitment and informed consent}

Eligible patients will be recruited from the existing patient population at the LUMC; this population from which the sample will be drawn is expected to be large enough to achieve adequate enrolment given the sample size needed. Patients who are interested in participating will receive a patient information letter and an informed consent form (documents available from CV on request). The information letter includes a statement that a decision not to participate in the study will not affect subsequent care. The coordinating investigator and an independent physician are available to answer any questions; their contact details are also provided. The patient will be contacted by the study team 5 days after receiving the information to discuss participation. Patients will only be included in the study after written informed consent has been obtained.

\section{Data storage and confidentiality}

All patient data will remain confidential and will not be shared outside the research team. Unique participant identifiers will be used to collect the data (except the informed consent form) and for entering data in a computerised database for the purposes of analysis. The identification code is kept separate and will only be accessible to the registered investigators. The investigators will safely store the informed consent forms together with the identification code (linking patient identifiers and personal details) for 15 years and will store the anonymised questionnaires (using participant codes as identifiers) separately for 15 years. The handling of the personal data will be carried out in compliance with the study protocol and the Dutch Personal Data Protection Act.

\section{Incentives}

The ephedrine and placebo tablets taken during the n-of- 1 trial will be provided by the sponsor at no cost to the patient. A patient undergoing treatment with ephedrine outside of this trial may have to pay or apply for reimbursement of the tablets through a leniency measure of the health insurance provider. Travel costs made by participants for hospital visits will be reimbursed.

\section{Trial-related injury}

The sponsor/investigator has obtained liability and clinical trial insurance which is in accordance with article 7 of the Dutch Medical Research Involving Human Subjects Act and the Measure regarding Compulsory Insurance for Clinical Research in Humans 2003, which provides cover for damage to research participants through injury or death caused by the study.

\section{Dissemination of results}

All patients will receive the results of their individual n-of-1 trials as per protocol (see 'Analysis' section). At the end of the study, the study team will finalise a report on the study. This report will be discussed with members of regulatory organisations involved in market approval and reimbursement of treatment. In addition, a lay summary of the overall findings of the study will be made available to the study participants. Anonymised results of the study will be published in a peer-reviewed journal, and will be presented at academic meetings and 
Table 1 WHO Trial Registration Data Set

Data category
Primary registry and trial
identifying number
Date of registration in primary
registry
Secondary identifiers
Source(s) of monetary or
material support
Primary sponsor
Secondary sponsor(s)
Contact for public queries

Information

EUCTR2014-001355-23-NL

27 March 2014

40960

The Netherlands Organisation for Health Research and Development (ZonMW), grant number 1520020301

Leiden University Medical Center

None

Ephedrine study contact

Leiden University Medical Center

Albinusdreef 2

2333 ZA Leiden

The Netherlands

Tel: (+31) 1715262118

Email: myasthenie@lumc.nl

Contact for scientific queries

Ephedrine study contact

Leiden University Medical Center

Albinusdreef 2

2333 ZA Leiden

The Netherlands

Tel: (+31) 1715262118

Email: myasthenie@lumc.nl

Public title

Scientific title

Country of recruitment

Condition studied

Intervention(s)

Key inclusion and exclusion criteria

Ephedrine as add-on therapy for patients with MG

Ephedrine as add-on therapy for patients with MG

Netherlands

MG

Active comparator: Ephedrine hydrochloride. Placebo comparator

Ages eligible for study: $\geq 18$ years

Sexes eligible for study: both

Inclusion criteria: Adult patient with generalised MG for whom treatment with pyridostigmine, either alone or in combination with low-dose prednisone or other immunosuppressive drugs, does not adequately improve symptoms

Exclusion criteria: purely ocular myasthenia, ephedrine is contraindicated or was not tolerated in the past, reliance on medium-to-high dose prednisone, intravenous immunoglobulin, or plasma exchange therapy, myasthenic crisis in past 3 months, thymectomy in past 6 months or scheduled, inability to comply with study procedures or give informed consent

\section{Study type}

\section{Interventional}

Controlled: yes, placebo

Allocation: randomised

Intervention model: multiple crossover assignment

Masking: double blind

Followed by optional open-label extension phase

7 October 2014

Date of first enrolment 4

Target sample size

Recruitment status

Primary outcome(s)

Complete as of 29 December 2014

Add-on effect of ephedrine on muscle strength/endurance as measured by the QMG score for all patients enrolled

Key secondary outcomes

Add-on effect of ephedrine for individual patients; feasibility of a larger series of n-of-1 trials, including patients' and physicians' experiences with the current trial; changes in secondary outcomes measures including the MG-Composite and MG-ADL; long-term effects of ephedrine (open-label extension study only); adverse effects of ephedrine

MG, myasthenia gravis; MG-ADL, MG-Activities of Daily Living; QMG, Quantitative Myasthenia Gravis.

scientific conferences. Only the registered investigators will have access to the individual patient data. Eligibility for authorship shall be in accordance with the ICMJE recommendations for authorship.

\section{DISCUSSION}

This series of randomised, controlled, double-blind n-of-1 trials with open-label extension phase will evaluate the effect of add-on treatment with ephedrine on 
muscle strength and endurance in patients with generalised, autoimmune MG. Series of n-of- 1 trials can be used to determine population treatment effectiveness as well as treatment effects in individual patients. N-of-1 trials may be particularly valuable to explore population treatment effects in patients with rare diseases, where parallel-group RCTs may be unfeasible due to small patient numbers. ${ }^{27} 28$

A limiting factor to the widespread use of series of n-of-1 trials, however, is the requirement for a stable disease course and for symptomatic, short-acting nature of the investigational treatment due to the crossover nature of the trial. ${ }^{43}$ Generalisability of the treatment effect to the entire population may also be limited if there is substantial heterogeneity in the study population treatment effect, especially if the sample size is small.

Despite these limitations, data from the current series of n-of-1 trials will be pooled to obtain a population treatment effect estimate, and will be used to facilitate regulatory innovation in two ways. First, the Dutch National Health Care Institute (NHCI) is in the process of implementing a 'feasible information trajectory' to obtain fitting evidence in situations where classic (group-)RCTs are not feasible. ${ }^{44}$ A workgroup of the NHCI will evaluate to what extent this study demonstrates that an n-of-1 trial design is suitable to generate evidence for reimbursement decisions. Second, based on the results of this trial, a workgroup of the Dutch Medicines Evaluation Board (MEB) will discuss scientific regulatory questions which should be clarified before evidence from pooled n-of- 1 trials could be used in a request for marketing authorisation. Furthermore, tailored implementation strategies, such as lectures and seminars, will be used to stimulate a range of stakeholders in the field of rare diseases to consider further development and use of n-of-1 trials.

Finally, we would like to invite clinicians and researchers in other hospitals to contact the study team if they are considering using (a similar version of) the current study protocol to conduct their own n-of-1 trials for add-on treatment with ephedrine in patients with generalised MG.

\author{
Author affiliations \\ ${ }^{1}$ Department of Epidemiology and Public Health, University College London, \\ London, UK \\ ${ }^{2}$ Department of Clinical Genetics, EMGO Institute for Health and Care \\ Research, VU University Medical Center, Amsterdam, The Netherlands \\ ${ }^{3}$ Department of Neurology, Leiden University Medical Center, Leiden, \\ The Netherlands \\ ${ }^{4}$ Department of Medical Statistics and Bioinformatics, Leiden University \\ Medical Center, Leiden, The Netherlands \\ ${ }^{5}$ Department of Clinical Pharmacy and Toxicology, Leiden University Medical \\ Center, Leiden, The Netherlands \\ ${ }^{6}$ Department of Care, Section Pharmaceutical Care, National Health Care \\ Institute, Diemen, The Netherlands \\ ${ }^{7}$ Department of Pharmacy, Radboud University Medical Center, Nijmegen, \\ The Netherlands
}

Acknowledgements The authors would like to acknowledge the members of the Medicines Evaluation Board Work Group; Dr Ilse Verstijnen (Dutch
National Health Care Institute); Johan Voerman, Marcel Timmen, and Dr Anja Horemans (Dutch Association for Neuromuscular Diseases); Dr Dirk Knol (VU University Medical Center); Professor Hans van Delden (University Medical Center Utrecht); and Professor Carin Uyl-de Groot (Erasmus University Rotterdam) for their feedback on the study protocol.

Contributors CV, SSW and JJGMV conceived of the study and initiated the design. AFL and CV wrote the study protocol with input from other authors. KJMS provided the investigational medicinal product information, designed the protocols for dispensing the investigational medicinal products, and provided the randomisation schedule. EWvZ provided statistical expertise, designed the statistical analysis, and will conduct the primary statistical analysis. MRK and YAH advised on alignment of the study protocol with the respective policies of the Dutch National Health Care Institute and the Dutch Medicines Evaluation Board. MCC provided feedback on the study protocol from the viewpoint of public health genomics. SSW and CV drafted the manuscript. All authors read and approved the final version.

Funding This work was supported by the Netherlands Organisation for Health Research and Development (ZonMW), grant number 1520020301. The trial is sponsored by Leiden University Medical Center.

Competing interests The employers of AFL, JJGMV, KJMS, MCC, SSW and CV were supported by a grant from the Netherlands Organisation for Health Research and Development (ZonMW) during the conduct of the study. SSW is an unpaid advisor for research and methodology for the Dutch Association for Neuromuscular Disorders since February 2013.

Ethics approval Medical Ethics Committee of Leiden University Medical Center.

Provenance and peer review Not commissioned; externally peer reviewed.

Data sharing statement Researchers interested in collaborations or further information are invited to contact CV at c.vrinten@ucl.ac.uk.

Open Access This is an Open Access article distributed in accordance with the Creative Commons Attribution Non Commercial (CC BY-NC 4.0) license, which permits others to distribute, remix, adapt, build upon this work noncommercially, and license their derivative works on different terms, provided the original work is properly cited and the use is non-commercial. See: http:// creativecommons.org/licenses/by-nc/4.0/

\section{REFERENCES}

1. Carr AS, Cardwell CR, McCarron PO, et al. A systematic review of population based epidemiological studies in myasthenia gravis. BMC Neurol 2010;10:46-54.

2. Juel VC, Massey JM. Myasthenia gravis. Orphanet J Rare Dis 2007;2:44-56

3. Meyer A, Levy Y. Geoepidemiology of myasthenia gravis. Autoimmun Rev 2010;9:A383-6.

4. Wirtz PW, Nijnuis MG, Sotodeh M, et al. The epidemiology of myasthenia gravis, Lambert-Eaton myasthenic syndrome and their associated tumours in the northern part of the province of South Holland. J Neurol 2003;250:698-701.

5. Buckley C. Autoimmune neurological diseases. Medicine 2008;36:653-7.

6. Keesey JC. Clinical evaluation and management of myasthenia gravis. Muscle Nerve 2004;29:484-505.

7. Mahadeva B, Phillips LH II, Juel VC. Autoimmune disorders of neuromuscular transmission. Semin Neurol 2008;28:212-27.

8. O'Neill GN. Acquired disorders of the neuromuscular junction Int Anesthesiol Clin 2006;44:107-21.

9. Skeie G, Apostolski S, Evoli A, et al. Guidelines for treatment of autoimmune neuromuscular transmission disorders. Eur J Neurol 2010;17:893-902

10. Electronic Medicines Compendium. Ephedrine hydrochloride. http:// www.medicines.org.uk/emc/ (accessed 8 Apr 2015).

11. Gallagher JP, Shinnick-Gallagher P. Ephedrine and neuromuscular transmission in vivo. Neuropharmacology 1979;18:749-54.

12. Milone M, Engel AG. Block of the endplate acetylcholine receptor channel by the sympathomimetic agents ephedrine, pseudoephedrine, and albuterol. Brain Res 1996;740:346-52.

13. Shinnick-Gallagher P, Gallagher JP. Ephedrine: a postsynaptic depressant drug at the mammalian neuromuscular junction. Neuropharmacology 1979;18:755-61. 
14. Sieb JP, Engel AG. Ephedrine: effects on neuromuscular transmission. Brain Res 1993;623:167-71.

15. Engel AG. The therapy of congenital myasthenic syndromes. Neurotherapeutics 2007;4:252-7.

16. Lashley D, Palace J, Jayawant S, et al. Ephedrine treatment in congenital myasthenic syndrome due to mutations in DOK7. Neurology 2010;74:1517-23.

17. Robb SA, Muntoni F, Simonds AK. Respiratory management of congenital myasthenic syndromes in childhood: Workshop 8th December 2009, UCL Institute of Neurology, London, UK. Neuromuscul Disord 2010;20:833-8.

18. Chaouch A, Beeson D, Hantaï D, et al. 186th ENMC international workshop: congenital myasthenic syndromes 24-26 June 2011, Naarden, The Netherlands. Neuromuscul Disord 2012;22:566-76.

19. Vrinten C, van der Zwaag AM, Weinreich SS, et al. Ephedrine for myasthenia gravis, neonatal myasthenia and the congenital myasthenic syndromes. Cochrane Database Syst Rev 2014;12: CD010028.

20. Hashimoto A, Hanada M, Okada S, et al. A case report of myasthenia gravis associated with Hashimoto's thyroiditis. Folia Psychiatr Neurol Jpn 1981;35:521-5.

21. Macdonald AM, Keen RI, Pugh ND. Myasthenia gravis and atracurium: a case report. Br J Anaesth 1984:56:651-4.

22. McAlpine JK, Thomson JE. Myasthenia gravis and Schmidt syndrome. Postgrad Med J 1988;64:787-8.

23. Zucker DR, Ruthazer R, Schmid $\mathrm{CH}$. Individual (N-of-1) trials can be combined to give population comparative treatment effect estimates: methodologic considerations. J Clin Epidemiol 2010;63:1312-23.

24. Nikles J, Mitchell GK, Schluter $P$, et al. Aggregating single patient (n-of-1) trials in populations where recruitment and retention was difficult: the case of palliative care. J Clin Epidemiol 2011;64:471-80.

25. Senior HE, Mitchell GK, Nikles J, et al. Using aggregated single patient (N-of-1) trials to determine the effectiveness of psychostimulants to reduce fatigue in advanced cancer patients: a rationale and protocol. BMC Palliat Care 2013;12:17-22.

26. Davis MP, Mitchell GK. Topics in research: structuring studies in palliative care. Curr Opin Support Palliat Care 2012;6:483-9.

27. Gupta S, Faughnan ME, Tomlinson GA, et al. A framework for applying unfamiliar trial designs in studies of rare diseases. J Clin Epidemiol 2011;64:1085-94.

28. Griggs RC, Batshaw M, Dunkle $M$, et al. Clinical research for rare disease: opportunities, challenges, and solutions. Mol Genet Metab 2009;96:20-6.

29. OCEBM Levels of Evidence Working Group. The Oxford 2011 Levels of Evidence. http://www.cebm.net/ (accessed 8 Apr 2015).
30. Burns TM, Conaway MR, Cutter GR, et al. Construction of an efficient evaluative instrument for myasthenia gravis: the MG Composite. Muscle Nerve 2008;38:1553-62.

31. Burns TM, Conaway M, Sanders DB, et al. The MG Composite: a valid and reliable outcome measure for myasthenia gravis. Neurology 2010;74:1434-40.

32. Wolfe Gl, Herbelin L, Nations SP, et al. Myasthenia gravis activities of daily living profile. Neurology 1999;52:1487-9.

33. Nikles CJ, Clavarino AM, Del Mar CB. Using n-of-1 trials as a clinical tool to improve prescribing. Br J Gen Pract 2005;55:175-80.

34. Scuffham PA, Nikles J, Mitchell GK, et al. Using N-of-1 trials to improve patient management and save costs. J Gen Intern Med 2010;25:906-13.

35. Nikles J, Mitchell GK, Clavarino A, et al. Stakeholders' views on the routine use of $n$-of- 1 trials to improve clinical care and to make resource allocation decisions for drug use. Aust Health Rev 2010;34:131-6.

36. Yelland MJ, Nikles CJ, McNairn N, et al. Celecoxib compared with sustained-release paracetamol for osteoarthritis: a series of $\mathrm{n}$-of-1 trials. Rheumatology (Oxford) 2007;46:135-40.

37. Howard JF Jr, Barohn RJ, Cutter GR, et al. A randomized, double-blind, placebo-controlled phase II study of eculizumab in patients with refractory generalized myasthenia gravis. Muscle Nerve 2013;48:76-84.

38. Tindall RS, Phillips JT, Rollins JA, et al. A clinical therapeutic trial of cyclosporine in myasthenia gravis. Ann N Y Acad Sci 1993;681:539-51.

39. Wolfe GI, Barohn RJ, Foster BM, et al. Randomized, controlled trial of intravenous immunoglobulin in myasthenia gravis. Muscle Nerve 2002;26:549-52.

40. Csajka C, Haller CA, Benowitz NL, et al. Mechanistic pharmacokinetic modelling of ephedrine, norephedrine and caffeine in healthy subjects. Br J Clin Pharmacol 2005;59:335-45.

41. Jaretzki A III, Barohn RJ, Ernstoff RM, et al. Myasthenia gravis: recommendations for clinical research standards. Task force of the medical scientific advisory board of the Myasthenia Gravis Foundation of America. Neurology 2000;55:16-23.

42. Benatar M, Sanders DB, Burns TM, et al. Recommendations for myasthenia gravis clinical trials. Muscle Nerve 2012;45:909-17.

43. Nikles J, Mitchell G, Walters $\mathrm{J}$, et al. Prioritising drugs for single patient (n-of-1) trials in palliative care. Palliat Med 2009;23: 623-34.

44. Heymans JM, Kleijnen S, Verstijnen IM. ['Fitting'evidence preferable when evaluating effectiveness of interventions]. Ned Tijdschr Geneeskd 2013;157:A5479. 